\title{
FINITELY CONVERGENT AND CONCENTRIC SEMIGROUPS
}

\author{
M. SATYANARAYANA
}

\begin{abstract}
In this paper we shall study Tamura's finitely convergent and concentric semigroups and provide some structure theorems. In particular commutative finitely convergent semigroups are completely characterized.
\end{abstract}

According to Tamura [3], a semigroup $S$ is said to be finitely convergent, if for every $x$ in $S, \cap_{n=1}^{\infty} S x^{n} S=S x^{m} S$ for some positive integer $m$. Simple semigroups, bands, nil semigroups, finite semigroups, right regular semigroups, left regular semigroups, and intraregular semigroups are finitely convergent semigroups. The bicyclic semigroup is an example of a regular semigroup which is finitely convergent. A free inverse semigroup with one generator is an example of a regular semigroup which is not finitely convergent. This example was furnished by Maria Szendrei in one of the seminar meetings in Szeged, while I was visiting Hungary. However we have

Proposition 1. If every right ideal is two-sided in a semisimple semigroup $S$ (in particular $S$ is a regular semigroup) then $S$ is finitely convergent.

Proof. Let $a \in S$. Then, by the characterization of semisimple semigroups in [2], $a \in S a S a S$. Since right ideals are two-sided, $S a S a S \subseteq a(S a) S \subseteq a^{2} S$ and $a \in a^{2} S$. So $S a S \subseteq S a^{2} S$ and $S a S=S a^{2} S$. Also $a \in a^{2} S$ implies $a^{2}=a . a \in a^{3} S$ and as before $S a^{2} S=S a^{3} S$. Then, by induction, $\cap_{n=1}^{\infty} S a^{n} S=S a S$ and hence $S$ is finitely convergent.

A semigroup $S$ is concentric if $\bigcap_{n=1}^{\infty} S x^{n} S=C(x)$ is constant for every $x$. This constant is called the closet of $S$. One can find many examples of concentric semigroups in [3]. A free semigroup generated by more than one element is one such example. Tamura [3] proved that a semigroup $S$ is concentric and finitely convergent iff $S$ is an ideal extension of a simple semigroup by a nil semigroup and thus in this case $S$ is semilattice-indecomposable. The question now arises whether the concentric property is necessary and sufficient for a finitely convergent semigroup to be semilattice-indecomposable, i.e., whether a finitely convergent semigroup which is semilattice-indecomposable is concentric. It is not true, since finite $o$-simple semigroups with zero-divisors are finitely convergent and semilattice-indecomposable but not concentric. Now we shall provide an external characterization and in some cases internal characterization of semilattice-indecomposable finitely

Received by the editors September 14, 1979.

AMS (MOS) subject classifications (1970). Primary 20M10.

Key words and phrases. Finitely convergent, concentric semigroups, closet. 
convergent semigroups. An ideal $A$ in $S$ is completely prime if $a b \in A$ implies either $a \in A$ or $b \in A$. It is well-known that a semigroup is semilattice-indecomposable iff $S$ has no completely prime ideals except itself.

For ready reference we shall quote the different characterizations of finitely convergent semigroups given by Tamura $[3$, p. 245] in the following

THEOREM 2. The following are equivalent on a semigroup $S$.

(i) $S$ is finitely convergent.

(ii) For every $a \in S$, there exists a natural number $n$ such that $a^{n} \in S a^{n+t} S$ for all $t>0$.

(iii) For every $a \in S, a^{n}$ is intraregular for some natural number.

(iv) For every $a$ in $S, a^{n} \in C(a)$ for some natural number $n$.

THEOREM 3. A finitely convergent semigroup $S$ is semilattice-indecomposable iff for every homomorphism $f$ of $S$ into the semigroup $N$ of nonnegative integers under multiplication, we have $f=0$ or $f(x)=1$ for every $x \in S$.

Proof. Let $S$ be semilattice-indecomposable. Suppose that $f$ is a homomorphism of $S$ into $N$. If $a \in S$, then $a^{n}=x a^{n+1} y$ for some natural number $n$ by Theorem 2 . Then $f\left(a^{n}\right)=f\left(x a^{n+1} y\right)$ and therefore $[f(a)]^{n}=f(x)[f(a)]^{n+1} f(y)$. Therefore $f(a)=$ 0 or $1=f(a) f(x y)$. The latter implies $f(a)=1$. If there exists an element $a$ in $S$ such that $f(a)=0$, then $f^{-1}(0)=\{x \in S: f(x)=0\}$ is a nonempty completely prime ideal. Since $S$ is semilattice-indecomposable, $f^{-1}(0)=S$ and thus $f=0$. Therefore from the preceding we have, $f(a)=1$ for every $a$ in $S$ if $f \neq 0$. Conversely let every $f$ have the prior property. If $S$ is not semilattice-indecomposable, then $S$ contains a completely prime ideal $P \neq S$. Define a function $f$ by $f(a)=0$ if $a \in P$ and $f(a)=1$ if $a \notin P$. Since $P$ is a completely prime ideal, $f$ is a homomorphism of $S$ into $N$, which is not true by hypothesis. Thus $S$ is semilatticeindecomposable.

REMARK. The above result is also true for any semisimple semigroup, which can be shown by adopting the same proof.

A semigroup $S$ is Archimedean if for every $x, y$ in $S, x^{n} \in S y S$ and $y^{m} \in S x S$ for some integers $n$ and $m$. An ideal $A$ in $S$ is called prime if $P$ and $Q$ are ideals in $S$ such that $P Q \subseteq A$, then either $P \subseteq A$ or $Q \subseteq A$. A semigroup $S$ is called $Q^{*}$-simple if $S$ has no proper prime ideals. Archimedean semigroups and $Q^{*}$-simple semigroups are always semilattice-indecomposable. We now provide an interval characterization for finitely convergent semigroups which belong to the previous two subclasses of semilattice-indecomposable semigroups. An element $x$ in $S$ is called intraregular if $x \in S x^{2} S$.

TheOREM 4. A finitely convergent semigroup is Archimedean iff $S$ contains a nonempty kernel $K$ and all the intraregular elements of $S$ belong to $K$. In this case $S$ is an ideal extension of a simple semigroup by a nil semigroup.

Proof. Let $S$ be Archimedean. By Theorem 2, $S$ contains intraregular elements. Let $a=x a^{2} y$ be an intraregular element. Then $a=x\left(x a^{2} y x a^{2} y\right) \cdot y \in x^{2} S y^{2}$. By induction, $a \in x^{n} S y^{n}$ for $n \geqslant 2$. Since $S$ is Archimedean, we can choose $n$ 
sufficiently large such that $x^{n}$ and $y^{n}$ belong to any given ideal $A$. Hence $a \in A$ and thus $a \in K$. Assume that the kernel $K$ be nonempty and every intraregular element is contained in $K$. Consider any two elements $x$ and $y$ in $S$. By Theorem 2, $x^{n}$ and $y^{m}$ are intraregular for some natural numbers $n$ and $m$. Therefore $x^{n} \in K$ $\subseteq S^{1} y S^{1}$ and $y^{m} \in K \subseteq S^{1} x S^{1}$ by hypothesis. Thus $S$ is Archimedean. Since for any $x \notin K$ and for any $y \in K, x^{n} \in S y S$ for some natural number $n, S$ is clearly an ideal extension of the simple semigroup $K$ by a nil semigroup.

For right duo semigroups (semigroups in which every right ideal is two-sided) and medial semigroups, the Archimedean property is equivalent to the semilatticeindecomposable property. Moreover by Theorem 4 [4, p. 777] a semigroup is a medial simple semigroup iff it is isomorphic to the direct product of an abelian group and a rectangular band. Hence we have

COROLlARY 5. If $S$ is a right duo or medial finitely convergent semigroup, then $S$ is semilattice-indecomposable iff the kernel $K$ of $S$ is nonempty and $K$ contains all intraregular elements. Moreover $S$ is an ideal extension of the simple semigroup $K$ by a nil semigroup. But in the medial case, $S$ is an ideal extension of the direct product of an abelian group and a rectangular band by a nil semigroup.

THEOREM 6. If $S$ is a $Q^{*}$-simple finitely convergent semigroup, then $S$ contains a nonempty kernel $K$ such that $S / K$ is a nil semigroup.

Proof. If $a \in S$, then by Theorem 2 there exists a natural number $m$ such that $a^{m} \in S a^{m+t} S$ for any $t \geqslant 0$. Call $a^{m}=x$. Let $T=\left\{x, x^{2}, \ldots\right\}$. Suppose there exists an ideal $A$ not containing $x$. Clearly $x \in \cap_{n=2}^{\infty} S x^{n} S$. Hence no power of $x$ belongs to $A$, i.e., $A \cap T=\varnothing$. Then by Zorn's lemma, there exists an ideal $B \supseteq A$ such that $B$ is maximal along all ideals containing $A$ with empty intersection with $T$. We claim that $B$ is a prime ideal. If $B$ is not a prime ideal, then there exist ideals $L$ and $M$ such that $L \subset B$ and $M \& B$ but $L M \subseteq B$. This implies that $x^{n} \in L \cup$ $B$ and $x^{m} \in M \cup B$ for some natural numbers $m$ and $n$ by the maximality of $B$. Therefore $x^{m+n} \in(L \cup B)(M \cup B) \subseteq B$, which is absurd. Hence $B$ is a prime ideal different from $S$, which again contradicts that $S$ is $Q^{*}$-simple. Thus we can assert that $x$ belongs to every ideal, so that the kernel $K$ is nonempty and $a^{m}=x \in K$. This implies $S / K$ is nil.

The prior discussion yields the following structure theorems.

TheOREM 7. Let $S$ be a medial semigroup. Then $S$ is finitely convergent iff $S$ is a semilattice of semigroups $S_{\alpha}$, each $S_{\alpha}$ being an ideal extension of a direct product of an abelian group and rectangular band by a nil semigroup.

Proof. By Corollary 2.13 of $\left[1\right.$, p. 32] $S$ is a semilattice of $N$-classes $S_{\alpha}$, where each $S_{\alpha}$ is semilattice-indecomposable. Now we claim that $S$ is finitely convergent iff each $S_{\alpha}$ is finitely convergent. Let $S$ be finitely convergent and $a \in S_{\alpha}$. Then $a^{m}=x a^{m+2} y$ for some natural number $m$ by Theorem 2. If $P$ is any completely prime ideal, then $x a$ or $a y \in P$ implies $a^{m} \in P$ and thus $a \in P$. Clearly $a \in P$ implies $x a$ and $a y$ belong to $P$. Hence $x a$ and $a y \in S_{\alpha}$, which implies that $S_{\alpha}$ is finitely convergent. The converse is obvious. Since $S$ is a medial semigroup, $S_{\alpha}$ is 
also a medial semigroup. Hence by Corollary $5, S_{\alpha}$ is an ideal extension of a direct product of an abelian group and a rectangular band by a nil semigroup. For the converse, it suffices to prove that if $S_{\alpha}$ is an ideal extension of a simple semigroup $A$ by a nil semigroup, then $S_{\alpha}$ is finitely convergent. If $a \in A$, then $a \in A a^{2} A$ and hence $a \in S_{\alpha} a^{2} S_{\alpha}$, which implies that $a$ is intraregular. If $a \notin A$, then $a^{n} \in A$ and therefore $a^{n}$ is intraregular as before. Thus $S_{\alpha}$ is finitely convergent by Theorem 2 .

COROllary 8. A commutative semigroup $S$ is finitely convergent iff $S$ is a semilattice of subsemigroups $S_{\alpha}$, which are ideal extensions of groups by nil semigroups or equivalently each $S_{\alpha}$ contains an idempotent.

In [3], Tamura reduced the study of concentric semigroups to that of concentric semigroups with zero or empty closet and left open the questions of determining the structure of latter semigroups. Now we describe these in terms of their ideal structure.

Proposition 9. Let $S$ be a concentric semigroup with empty closet (or zero closet if $S$ contains 0$)$. Then the following are true.

(i) For every $x(\neq 0)$ in $S, x \notin S x, x \notin x S$ and $x \notin S x S$. No (nonzero) element is right regular, left regular, intraregular, regular and an idempotent.

(ii) If $S=S^{2}$, then $S$ has no maximal left or right or two-sided ideals. If $S \neq S^{2}$, then $S$ contains maximal left, right and two-sided ideals; $S^{2}$ is the intersection of all maximal left ideals, intersection of all maximal right ideals and also maximal two-sided ideals. Every maximal one-sided ideal is two-sided and its complement consists of a single element.

(iii) Every ideal $A$ in $S$ is also concentric with empty (or zero) closet.

(iv) If $S$ does not contain 0 , then $S$ has no maximal one-sided or two-sided ideals. If $S$ contains 0 , then every minimal one-sided or two-sided ideal $A$ satisfies the property $A^{2}=0$.

Proof. (i) If $x \in S x S$, then $x=s_{1} x t_{1}$ for some $s_{1}$ and $t_{1}$ in $S$ and thus $x=s_{1}^{2} x t_{1}^{2} \in S s_{1} S$. By induction, we have $x \in S s_{1}^{n} S=\varnothing$ (or zero). Hence $x \notin$ $S x S$. The other conclusions can be proved in a similar fashion.

(ii) If $M$ is a maximal left ideal, then there exists a nonzero $x$ in $M$. So $S=M \cup S^{1} x$. If $S=S^{2}$, then $S=S^{2}=M \cup S x$ and hence $x \in S x$, which is a contradiction by (i). Thus $S$ contains no maximal left ideals. Similarly we can prove that $S$ contains no maximal right and two-sided ideals. If $S \neq S^{2}$, clearly for any $x \in S \backslash S^{2}, S \backslash x$ is a maximal one-sided and also two-sided ideal. Let $S \neq S^{2}$ and $L$ be the intersection of all maximal left ideals. If $t \in L \backslash S^{2}$, then $S \backslash t$ is a maximal left ideal and hence contains $t$. This contradiction proves $L \subseteq S^{2}$. If $t \in S^{2} \backslash L$, then $S=M \cup S^{1} t$ for some maximal left ideal $M$ which does not contain $t$. But $S^{2}=S M \cup S^{2} t \cup S t$, which implies $t \in S t$, which is not true by (i). Hence $S^{2} \subseteq L$ and therefore $L=S^{2}$. Similar proof yields that $S^{2}$ is the intersection of all maximal right ideals and also the intersection of all maximal ideals. To show that the complement of every maximal left ideal consists of a single element, let us assume that there exists a maximal left ideal $M$ not containing two distinct 
elements $x$ and $y$. Then $S=M \cup x \cup S x=M \cup y \cup S y$. Hence $x \in S y \subseteq S x$, which is not true by (i). Similar proof can be given in the case of maximal right ideals. Every maximal left ideal $M$ is two-sided since $M$ is of the form $S \backslash x$ for some $x \in S \backslash S^{2}$ and thus if $m x \notin M$ for some $m$ in $M$, then $x=m x$, which is not true by (i) and the proof for maximal right ideals is similar.

(iii) If $A$ is an ideal of $S$, then $x \in \cap_{n=1}^{\infty} A a^{n} A$ for $a \in A$ implies $x \in S a^{n} S$ for every natural number $n$. Hence the conclusion is evident.

(iv) It suffices to prove the results for minimal left ideals since similar proof can be given in other cases. If $S$ does not contain 0 and if $A$ is a minimal left ideal, then for any $x \in A, x \in A=S x$, which is not true by (i). Let $S$ contain 0 and $A$ be a minimal left ideal. If $A^{2} \neq 0$, then $A^{2}=A$ by minimality of $A$. If $x \in A$ and $x \neq 0$, then $x \cup S x \neq 0$, so that $A=x \cup S x$. Hence $x \in(x \cup S x)(x \cup S x)$, which implies $x \in S x$, contradicting (i).

THEOREM 10. Let $S$ be a semigroup which is not simple (or 0-simple if $S$ contains 0 ). Then $S$ is a concentric semigroup with empty (or zero) closet iff $S$ is one of the following.

(i) $S$ is a globally idempotent semigroup with no maximal ideals such that every proper ideal is a concentric subsemigroup with empty (or zero) closet.

(ii) $S$ is a nonglobally idempotent semigroup such that $S^{2}$ is a concentric semigroup with empty (or zero) closet.

Proof. If $S$ is a concentric semigroup with empty (or zero) closet, then $S$ is one of the previously prescribed forms by Proposition 9. Conversely, let $S$ be the form (i). Suppose that $x \in S$ and $y \in \cap_{n=1} S x^{n} S$. Since $S$ is not simple (or 0-simple) and since $S$ contains no maximal ideals, $S=\cup A_{\alpha}$, where $\left\{A_{\alpha}\right\}$ is the set of all proper ideals. Hence $x$ belongs to some $A_{\alpha}$. Now $y \in S x x^{n} x S \subseteq A_{\alpha} x^{n} A_{\alpha}$. Since $A_{\alpha}$ is a concentric semigroup with empty (or zero) closet, either the prior fact is inadmissible or $y=0$ if $S$ contains 0 . Thus $S$ is a concentric semigroup with empty (or zero) closet. Suppose now that $S$ is of the form (ii). Let $x \in S$. Then $y \in \cap{ }_{n=1}^{\infty} S x^{n} S$ implies $y \in S^{2}\left(x^{2}\right)^{n} S^{2}$ for every natural number $n$. Since $S^{2}$ is concentric with empty (or zero) closet, $\cap_{n=1}^{\infty} S x^{n} S=\varnothing$ (or zero). Thus the conclusion is evident.

\section{REFERENCES}

1. M. Petrich, Introduction to semigroups, Merrill, Columbus, Ohio, 1973.

2. M. Satyanarayana, On a class of semisimple semigroups, Semigroup Forum 10 (1975), 129-138.

3. T. Tamura, The study of closets and free contents related to semilattice decompositions of semigroups, Semigroups (Proc. Sympos., Wayne State Univ., Detroit, Mich., 1968), Academic Press, New York, 1969, pp. 221-260.

4. _, Notes on medial Archimedean semigroups without idempotents, Proc. Japan Acad. 44 (1968), 776-778.

Department of Mathematics, Bowling Green State University, Bowling Green, Ohio 43403 